'Unidad de Medicina Física y Rehabilitación. Clínica Universidad de los Andes. Santiago, Chile.

${ }^{2}$ Servicio de Medicina Física y Rehabilitación, Clínica Indisa. Santiago, Chile. ${ }^{3}$ Centro de Nutrición y Bariátrica, Clínica Las Condes. Santiago, Chile.

${ }^{4}$ Servicio de Cirugía Bariátrica y Metabólica, Clínica Santa María. Santiago, Chile.

${ }^{5}$ Centro de Tratamiento de la Obesidad, Red Salud UC-Christus. Santiago, Chile. ${ }^{a}$ Kinesióloga. ${ }^{\text {b} N u t r i o ́ l o g a . ~}$

${ }^{c}$ Residente Medicina del Deporte. Universidad Mayor. Santiago, Chile. ¿Kinesiólogo.

Contribución de los autores: Escritores y revisores significativos del escrito LG y JC; concepto y diseño LG, JC, JP; escritores y revisores de la sección de ejercicio aeróbico LG y JC; escritores y revisores de la sección de ejercicio de fuerza ET; escritores y revisores de la sección de suplementación proteica JP, MO, MJE; revisor de la sección de suplementación proteica AR; escritores y revisores de la sección de actividad física IP y MV.

Trabajo Financiado por Sociedad Chilena de Cirugía Bariátrica y Metabólica (SCCBM).

Recibido el 22 de octubre de 2018, aceptado el 11 de noviembre de 2019.

Correspondencia a: Luis González

Nevería 444 Las Condes, Santiago (piso 5). rojas.gonzalez.luis@gmail.com

\section{Recomendaciones de actividad física y ejercicio en el paciente adulto sometido a cirugía bariátrica. Documento de Consenso}

\author{
JAVIERA CANCINO-RAMÍREZ ${ }^{1, \mathrm{a}}$, ERIKA J. TRONCOSO-ORTIZ ${ }^{2, \mathrm{a}}$, \\ JOHANNA PINO ${ }^{3, a}$, MARIELA OLIVARES ${ }^{3, a}$, \\ MARÍA JOSÉ ESCAFFI ${ }^{3, b}$, ANTONIETA RIFFO $^{c}$, \\ ISRAEL PODESTÁ D..$^{4, d}$, MANUEL VÁSQUEZ M. ${ }^{4, d}$, \\ LUIS GONZÁLEZ-ROJAS ${ }^{5, \mathrm{~d}}$
}

\section{Exercise and physical activity in adults who underwent bariatric surgery. Consensus document}

Physical exercise is useful in people who underwent bariatric surgery. However, the right dosage is still a topic for discussion. The aim of this article is to consolidate the prescription criteria for physical activity and exercise in bariatric patients. A panel of experts to whom the topics were previously assigned for review, met to reach a consensus. Each topic was presented and subjected to discussion and voting by the participants and attendants who were exercise professionals from different obesity treatment centers. We report the conclusions reached for aerobic exercise, strength training, protein supplementation and physical activity for weight maintenance in bariatric patients.

(Rev Med Chile 2019; 147: 1468-1486)

Key words: Bariatric Surgery; Exercise; Obesity.
L a obesidad ha aumentado drásticamente en las últimas décadas a nivel global ${ }^{1,2}$. El tratamiento más eficaz en el control del peso ha demostrado ser la cirugía bariátrica, lo que en conjunto con la disminución en las complicaciones asociadas ha traído consigo un incremento en su realización ${ }^{2}$. A su vez, el tratamiento multidisciplinario en pacientes bariátricos potencia sus resultados y promueve la mantención de éstos en el largo plazo ${ }^{3,4}$.

Diversos estudios han propuesto que la actividad física podría mejorar la calidad y aumentar la baja de peso producto de la cirugía ${ }^{3,5,6}$, favorecer la mantención del peso en el tiempo ${ }^{4} \mathrm{y}$ aumentar su rol regulatorio en alteraciones del metabolismo de la glucosa ${ }^{3}$.

A raíz de los beneficios potenciales y crecientes del ejercicio en el paciente bariátrico es que nace la necesidad de la Sociedad Chilena de Cirugía Bariátrica y Metabólica por crear un área especializada en salud y ejercicio físico en estos pacientes.

El aumento exponencial de la literatura científica y de los centros multidisciplinarios que realizan cirugía bariátrica a nivel nacional conlleva al incremento de profesionales del ejercicio enfrentados a este perfil de pacientes. A partir de esto, una de las primeras funciones que el área de salud y ejercicio físico ha considerado relevante 
es la generación de un documento de consenso buscando unificar los criterios mínimos de tratamiento a nivel nacional.

\section{Método}

Se seleccionaron 9 expertos: académicos universitarios, investigadores activos en el tema y/o miembros destacados de sociedades científicas que fueron asignados a grupos de investigación independientes para realizar revisiones bibliográficas de diferentes áreas. Los temas a revisar fueron previamente establecidos por el equipo de expertos. Estas revisiones fueron presentadas, discutidas y llevadas a consenso en el primer encuentro de Kinesiología en la Obesidad y Cirugía Bariátrica realizado el día 19 de noviembre de 2016. Finalmente, cada grupo actualizó la literatura correspondiente para la elaboración del presente escrito.

Se realizaron búsquedas en Medline (Pubmed) para cada área de interés, acorde a los principios establecidos en el Manual Cochrane de revisiones sistemáticas de intervenciones ${ }^{7}$. La búsqueda se realizó sobre publicaciones históricas, efectuada a fecha del 22 de marzo de 2018 y los criterios de selección de los artículos están detallados en la Tabla 1. Las áreas de interés acordadas por la directiva para la revisión bibliográfica fueron las siguientes:

1) Evaluación y prescripción del ejercicio aeróbico en el paciente bariátrico.

2) Evaluación y prescripción del ejercicio de sobrecarga en el paciente bariátrico.

3) Suplementación proteica para aminorar la pérdida de masa muscular en el paciente bariátrico.

4) Actividad física y mantención del peso en el paciente bariátrico.

\section{Revisión de artículos y extracción de datos}

La revisión de los resultados de cada búsqueda fue realizada mediante una aproximación sistemática desarrollada por 2 autores diferentes. Cada autor analizó los títulos, luego los resúmenes y, finalmente, el texto completo de forma independiente. Los conflictos en cuanto a la selección de estudios fueron resueltos bajo común acuerdo $\mathrm{y}$, en caso de no lograrlo, fueron sometidos a la revisión y criterio de un tercer autor. Ambos autores extrajeron los datos de los artículos que cumplían los criterios de selección, y fueron revisados y confirmados en conjunto de común acuerdo.

\section{Discusión}

\section{Ejercicio aeróbico en el paciente bariátrico}

\section{Evaluación de la capacidad cardiorrespiratoria}

En el paciente bariátrico, el objetivo de la evaluación es determinar la capacidad cardiorrespiratoria a través de una prueba de estrés cardiopulmonar que nos permita prescribir el ejercicio aeróbico y evaluar la respuesta al entrenamiento, teniendo en consideración sus experiencias previas habitualmente negativas relacionadas con el ejercicio $y / o$ actividad física ${ }^{8}$. En la literatura revisada en la Tabla $2^{6,9-18}$, la metodología más utilizadas es la evaluación directa del consumo máximo $\left(\mathrm{VO}_{2 \max }\right)$ o pico de oxígeno mediante ergoespirometría, utilizando protocolos escalonados con duración de 5-12 min. Dentro de las evaluaciones indirectas la realización del protocolo de Astrand o Bruce puede entregar una estimación del $\mathrm{VO}_{2 \max }{ }^{19}$ y parámetros de carga interna y/o externa para la prescripción del ejercicio.

\section{Recomendaciones}

\section{Pre-cirugía}

- Se debe realizar alguno de los protocolos mencionados previamente hasta el agotamiento (considerando el riesgo cardiovascular del paciente) obteniendo idealmente frecuencia cardiaca máxima, watts, frecuencia cardiaca de reserva y/o METS.

\section{Post-cirugía}

- Dada la poca tolerancia a ejercicios de alta intensidad en la etapa aguda, la realización de protocolos hasta el agotamiento debe ser desde los 3 meses en adelante. No existen artículos que hayan descrito la variación de la capacidad cardiorrespiratoria previo a ese período, por lo que su valor en la evaluación o prescripción se encuentra aún en discusión.

\section{Prescripción de ejercicio aeróbico}

El objetivo de la prescripción de ejercicio aeróbico es entregar al paciente parámetros de dosificación individualizados y atingentes a su 


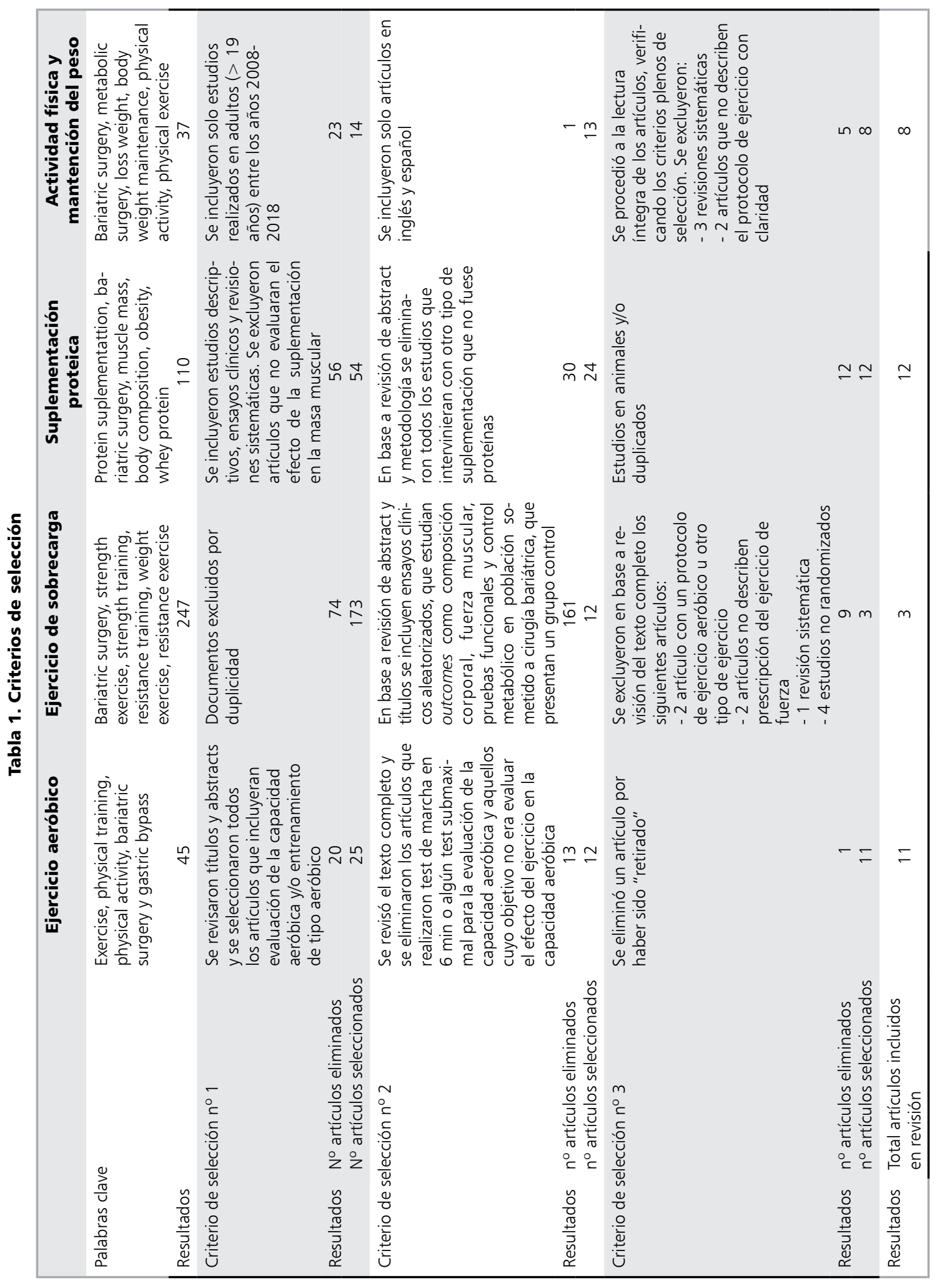


cuadro clínico para optimizar el entrenamiento, buscando potenciar y complementar los resultados de la cirugía bariátrica. Es importante comenzar la intervención de manera precoz, idealmente previo a la cirugía ya que esto ha demostrado una mejoría en la condición física, en la adquisición de un estilo de vida activo y podría favorecer la baja de peso ${ }^{20}$.

En la literatura disponible el ejercicio aeróbico ha generado incrementos significativos de la capacidad cardiorrespiratoria en esta población $n^{9-11,14,18}$. En dichas intervenciones el tiempo de inicio fluctuó entre 1 y 3 meses post cirugía. La frecuencia de las sesiones de ejercicio fue de 3-5 veces por semana. El tiempo promedio de entrenamiento inicialmente fue de 30-45 min hasta alcanzar 120 min semanales a los 3 meses post cirugía. La intensidad de trabajo fue moderada y se determinó principalmente mediante la frecuencia cardiaca máxima teórica (FCMT) (60-70\%), frecuencia cardíaca de reserva (FCR) (55-85\%) y en algunas ocasiones según la escala de Borg ${ }^{12-14}$ o $\mathrm{Vo}_{2 \max }$ (70\%). Todos realizaron modalidades de ejercicio mono estructurales como caminatas o treadmill, cicloergómetro, remo, elíptica o cicloergómetro de brazos (Tabla 2).

\section{Recomendaciones}

\section{Pre-cirugía}

- Se debe realizar ejercicio aeróbico de intensidad moderada (40-60\% FCR) 3-5 veces por semana sumando un total de 150 min semanales.

\section{Post-cirugía}

- Recomendamos la utilización de la FCR debido a una posible disminución en la actividad del sistema nervioso simpático post cirugía bariátrica descrita por algunos autores ${ }^{21}$. Para el cálculo se sugiere utilizar la FCM alcanzada en la evaluación previa a la cirugía, siempre que la prueba haya sido hasta el agotamiento, de no ser así se debe utilizar la FCMT según Tanaka y cols ${ }^{22}$.

- Semanas 2 a 4: Desde el inicio del régimen alimentario de papillas se debe realizar ejercicio aeróbico de intensidad moderada (40-60\% de la FCR), 3 veces por semana sumando un total de 90 min semanales.

- Semanas 4 a 12: La intensidad se mantiene, sin embargo, se debe progresar hasta alcanzar los volúmenes previos a la cirugía (150 min/ semana), considerando la tolerancia de cada paciente. Esto puede lograrse mediante el incremento de la frecuencia semanal y/o del tiempo por sesión.

- Semanas 12-24: Se permite la inclusión de actividades lúdicas, deportivas y/o también de ejercicios en intervalos de alta intensidad.

- 6 meses en adelante: Se debe aumentar el tiempo a $200 \mathrm{~min} / \mathrm{semanales,} \mathrm{manteniendo}$ las indicaciones de la etapa anterior ${ }^{23}$.

\section{Ejercicio de fuerza en el paciente bariátrico}

\section{Evaluación de la fuerza muscular}

En el paciente sometido a cirugía bariátrica es necesaria la evaluación de la fuerza muscular para poder prescribir el ejercicio de fortalecimiento y evaluar la respuesta en las distintas etapas post cirugía.

En la literatura analizada en la Tabla 3, Daniels y cols. ${ }^{24}$ evaluaron la fuerza muscular mediante una repetición máxima (1RM) de acuerdo a la recomendación del Colegio Americano de Medicina del Deporte (ACSM) de 201325. Hassannejad y cols. ${ }^{6}$ estimaron 1RM de tren superior de manera indirecta utilizando las ecuaciones propuestas por Desgorces y cols. ${ }^{26}$, sin embargo, esta ecuación se ha validado en atletas y su uso no se ha extendido ni validado en sujetos sedentarios o con exceso de peso. Por otro lado, las ecuaciones de Brzycki y de Epley-Welday han sido ampliamente utilizadas en población con obesidad ${ }^{27,28}$.

En la literatura seleccionada, para la evaluación funcional de tren inferior se utilizaron adaptaciones de la prueba de pararse y sentarse ${ }^{6,17}$ y en tren superior se realizó la prueba de dinamometría de mano para determinar la fuerza de prensión ${ }^{6}$.

\section{Recomendaciones}

\section{Pre-cirugía}

- Considerando el contexto del paciente, se recomienda la estimación de 1RM de acuerdo a las fórmulas propuestas por Brzycki (si se realizarán menos de 10 repeticiones) y la de Epley-Welday (si se realizarán más de 10 repeticiones) que han sido ampliamente utilizadas en población con obesidad ${ }^{27,28}$.

- Se deben realizar no más de 5 intentos por cada ejercicio y se deben dejar 1 a 2 min de descanso entre cada intento ${ }^{6}$. 


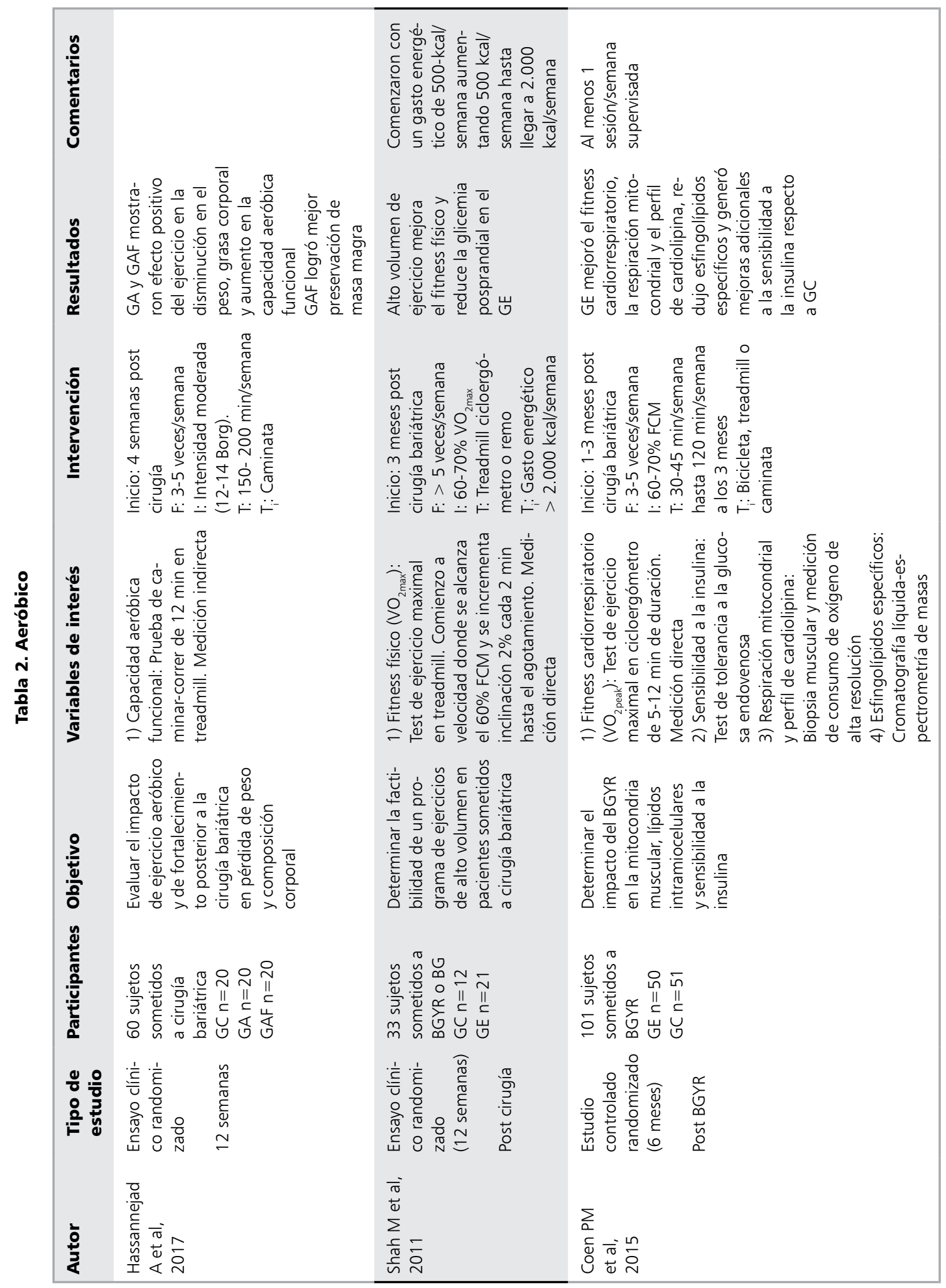




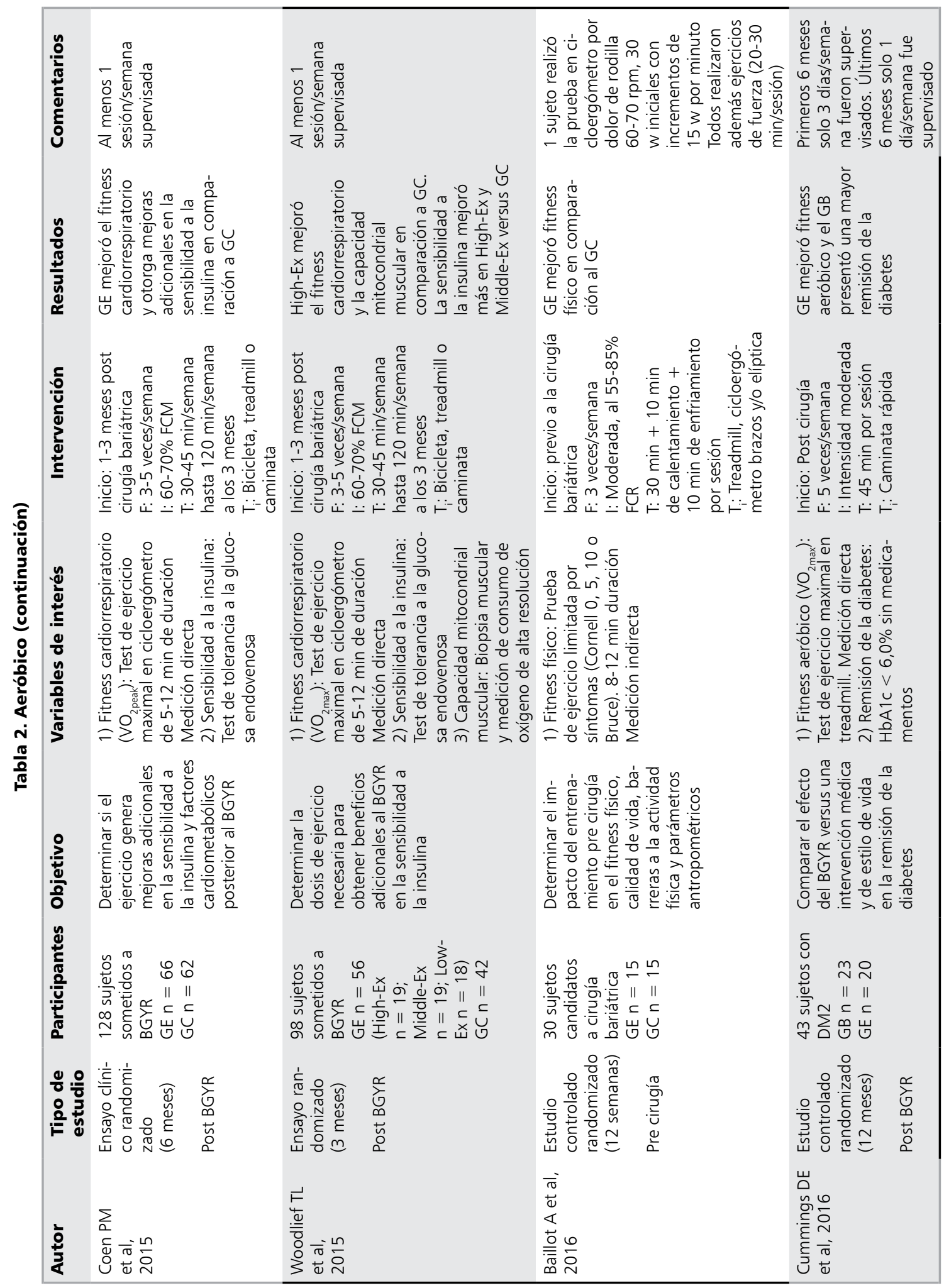




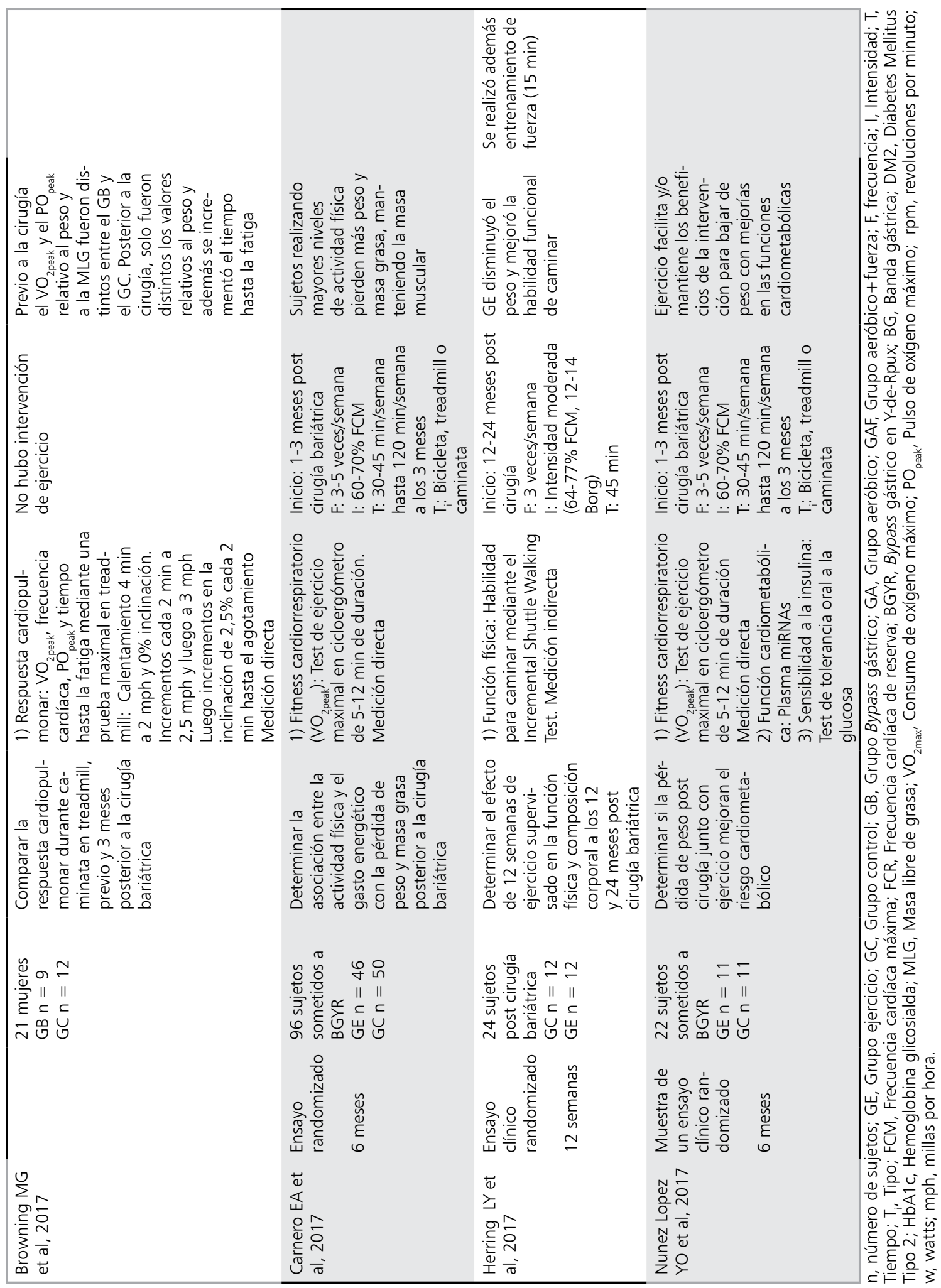




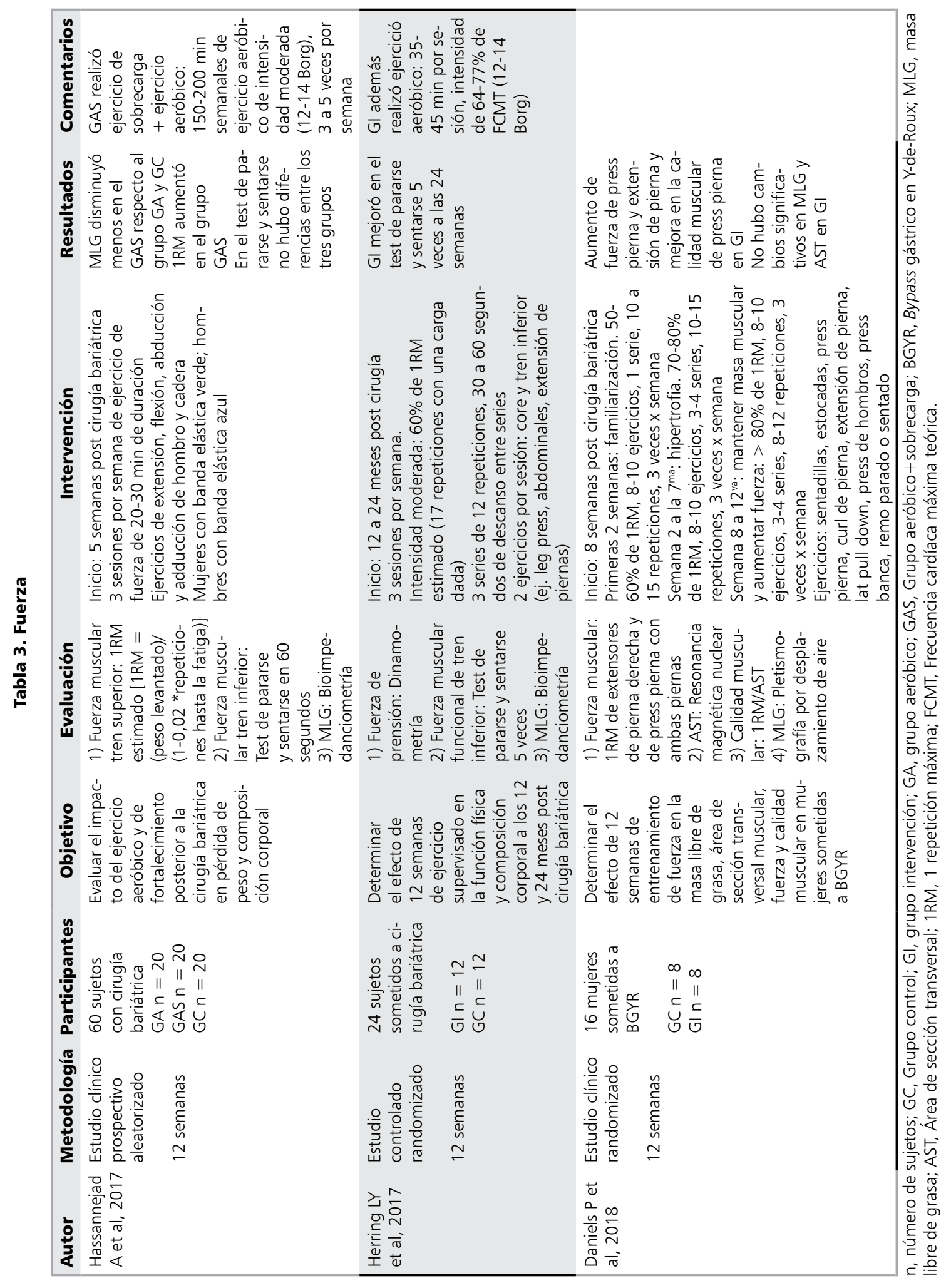


- Al menos, se debe evaluar extensión de rodilla, prensa de pierna y prensa de pecho. En caso de no contar con la implementación necesaria, se puede considerar la realización de pruebas funcionales como la prueba de pararse y sen$\operatorname{tarse}^{6,17}$.

- Se aconseja la evaluación de la fuerza de prensión dada su importancia como indicador de salud $^{29}$, sin embargo, no se ha evidenciado su utilización en la prescripción del ejercicio.

\section{Post-cirugía}

- Debido a la menor tolerancia al ejercicio de alta intensidad que presentan estos pacientes en el período post quirúrgico agudo, no se recomienda la evaluación de fuerza hasta la extenuación previo a los 3 meses post cirugía. Posterior a este período la evaluación debe guiarse por las recomendaciones pre-quirúrgicas.

\section{Prescripción del ejercicio de fuerza}

El objetivo de prescribir ejercicio de fuerza en el paciente sometido a cirugía bariátrica es disminuir la pérdida de masa libre de grasa posterior a la cirugía. Esto dado que dicha pérdida se asocia con implicancias negativas en la movilidad, función física y en la reganancia de peso ${ }^{30,31}$.

En los artículos seleccionados (Tabla 3) el entrenamiento de fuerza comenzó entre la quinta y la octava semana post cirugía, la frecuencia de los entrenamientos fue de 3 veces por semana comenzando entre $50-60 \% 1 \mathrm{RM}$ y progresando a más de $80 \% 1 \mathrm{RM}$ a la octava semana de entrenamiento. Se realizaron ejercicios de grandes grupos musculares, entre 8-10 ejercicios por sesión en 3 series de 10-15 repeticiones progresando a 3-4 series de 8-12 repeticiones cuando el peso supera el $80 \%$ de 1RM. Los ejercicios realizados fueron ejercicios con máquinas como prensa de piernas, extensión de rodillas, prensa de pecho, remo sentado, prensa de hombro, jalón de polea alta, entre otros y ejercicios con el peso corporal como: sentadillas, estocadas y ejercicios de flexión, extensión, abducción y aducción de hombro y cadera (con banda elástica), además de ejercicios abdominales (CORE). En dichos estudios se consideraron descansos de 30-60 segundos entre series.

En la búsqueda bibliográfica no se encontraron intervenciones pre-quirúrgicas que cumplieran con las condiciones mencionadas, sin embargo, esta fase tiene muchas semejanzas con el tratamiento conservador del paciente con obesidad, buscando disminuir el peso del paciente a través de modificaciones en su alimentación ${ }^{32}$. Bajo este contexto el ejercicio de fuerza ha evidenciado un rol fundamental en la mantención de la masa magra ${ }^{33}$.

\section{Recomendaciones}

\section{Pre-cirugía}

- Se debe comenzar con una etapa de familiarización de los ejercicios con un peso entre 40 a $60 \%$ de 1 RM y realizando solo una serie de 10 a 15 repeticiones, enseñando un correcto gesto de ejecución y evitando maniobras de valsalva.

- Luego se debe aumentar progresivamente el peso hasta sobrepasar $80 \%$ de $1 \mathrm{RM}$ y se aumenta el número de series a 3-4 series de 8-12 repeticiones, con 30-60 segundos de descanso entre cada serie. En caso que por metodología no se pueda prescribir en base al porcentaje de $1 \mathrm{RM}$, el ejercicio hasta el fallo muscular puede ser una alternativa, con las consideraciones pertinentes para este perfil de pacientes.

- Se recomienda la realización de 8-10 ejercicios de grandes grupos musculares que deben ir incorporándose de forma progresiva.

\section{Post-cirugía}

- El inicio del entrenamiento de fuerza debe ser precoz (durante el primer mes post cirugía, posterior al inicio del régimen de papillas) con una frecuencia de 3 veces por semana, que debe ser prescrito en base a la evaluación de 1RM realizada previo a la cirugía.

- La progresión debe ser similar a las recomendaciones pre-quirúrgicas, comenzando siempre con cargas menores que las utilizadas previo a la cirugía.

- Los ejercicios de la musculatura abdominal se deben incorporar al mes post-cirugía y se sugiere optar por ejercicios de tipo isométrico en una primera fase. Se recomienda incluir ejercicios de control postural y estabilización lumbo-pélvica.

\section{Suplementación proteica para aminorar la pérdida de masa muscular en el paciente bariátrico}

La suplementación proteica en los pacientes sometidos a cirugía bariátrica cobra relevancia dado que éstos tienden a consumir un bajo 
aporte de proteínas en la dieta ${ }^{34}$. De acuerdo a la literatura revisada en la Tabla $4^{35-46}$ el objetivo de la suplementación proteica es alcanzar las recomendaciones de ingesta diaria de proteínas $(0,8-1,2 \mathrm{~g} / \mathrm{kilo} \text { de peso ideal })^{34}$, lo que ha mostrado favorecer la preservación de masa muscular o masa magra ${ }^{43,45}$ e incluso su aumento posterior a la cirugía ${ }^{44,46}$. La proteína de suero de leche es la proteína de mayor calidad biológica conocida dada la gran cantidad de aminoácidos de cadena ramificada que posee (valina, leucina, isoleucina), los que se relacionan con la síntesis y reparación muscular $^{47-49}$. En sujetos sanos y activos, al comparar la síntesis de masa muscular posterior al entrenamiento de fuerza con suplementación de proteína de soya, caseína y proteína de suero de leche, los mejores resultados se obtienen con $22 \mathrm{~g}$ de proteína de suero de leche inmediatamente después de realizar ejercicio ${ }^{36,50}$. No está determinada la cantidad exacta de suplemento de proteína recomendada, sin embargo, en los estudios analizados la suplementación fue de 0,5-1 g por kilo de peso ideal, coincidiendo en una dosis mínima de 30-35 g/día.

\section{Recomendaciones $^{*}$}

- La ingesta mínima de proteínas post-cirugía bariátrica debe ser de 0,8-1,2 g de proteínas por kilo de peso ideal.

- Se aconseja comenzar con suplementación de proteína de suero de leche en polvo a los 7 días post-cirugía, con una dosis de 25-30 g al día, ajustándose a cada persona según adherencia al plan alimentario indicado.

- Los días que se realice ejercicio se debe incorporar un batido adicional de $20 \mathrm{~g}$ de proteínas disuelto en 100-200 cc de agua, según tolerancia.

- La ingesta debe ser inmediatamente antes y/o después de la sesión de ejercicio.

${ }^{\star}$ Todo lo expuesto anteriormente queda supeditado a la tolerancia del paciente y sujeto a la decisión del equipo tratante respecto del manejo individual de cada paciente.

\section{Actividad física y mantención del peso en el paciente sometido a cirugía bariátrica}

El paciente que se somete a cirugía bariátrica se caracteriza por tener un comportamiento más sedentario que sujetos con normopeso ${ }^{23,51} \mathrm{e} \mathrm{inclu-}$ so que aquellos sujetos con obesidad que no son sometidos a cirugía bariátrica ${ }^{52,53}$. En el paciente bariátrico, la adherencia a los cambios en el estilo de vida es esencial para el mantenimiento a largo plazo de la pérdida de peso posterior a la cirugía bariátrica $^{54,55}$. Sin embargo, a pesar de incrementar sus niveles de actividad física no logran alcanzar las recomendaciones internacionales de las guías de salud ${ }^{56-59}$.

Los niveles de actividad física previo a la cirugía se relacionan con el éxito en la pérdida de peso y con los niveles de actividad física post-cirugía ${ }^{57,58,60}$. Por otro lado, múltiples estudios muestran que la práctica de actividad física ${ }^{17,58,59,61}$, el tiempo sedente y el comportamiento sedentario post-cirugía $^{58}$ se correlacionan de manera positiva con la pérdida y más aún con la mantención del peso a largo plazo. Además, existe una correlación entre una menor cantidad de pasos diarios y un bajo porcentaje de pérdida de peso en el proceso quirúrgico $^{62}$. También se ha visto que la actividad física en tiempo libre previo a la cirugía es un predictor independiente del porcentaje de peso perdido a los 12 meses post-cirugía ${ }^{62}$. Entonces, el objetivo de evaluar los niveles de actividad física y el comportamiento sedentario es poder desarrollar intervenciones y planes de tratamiento a largo plazo orientados a favorecer la baja y la mantención del peso en esta población.

Según lo revisado en la Tabla $5^{17,23,58-63}$, la evaluación de los niveles de actividad física se realizó mediante acelerometría, conteo de pasos con podómetro y/o mediante cuestionarios o entrevistas. En base a dichas mediciones se puede categorizar al sujeto como activo e inactivo. Además, algunas guías clínicas recomiendan acumular entre 150 y $300 \mathrm{~min}$ de ejercicio de intensidad moderada a la semana o un promedio de al menos 10.000 pasos diarios para mantener la pérdida de peso o minimizar la reganancia en el largo plazo ${ }^{64-67}$.

\section{Recomendaciones}

- La evaluación debe ser mediante el cuestionario IPAQ en su forma corta para obtener el valor de METs/semana de actividad física y el tiempo sedentario en h/día.

- Se sugiere la utilización de cuenta pasos para favorecer el gasto energético no asociado a ejercicio físico, con una referencia de 10.000 pasos día ${ }^{68}$. 


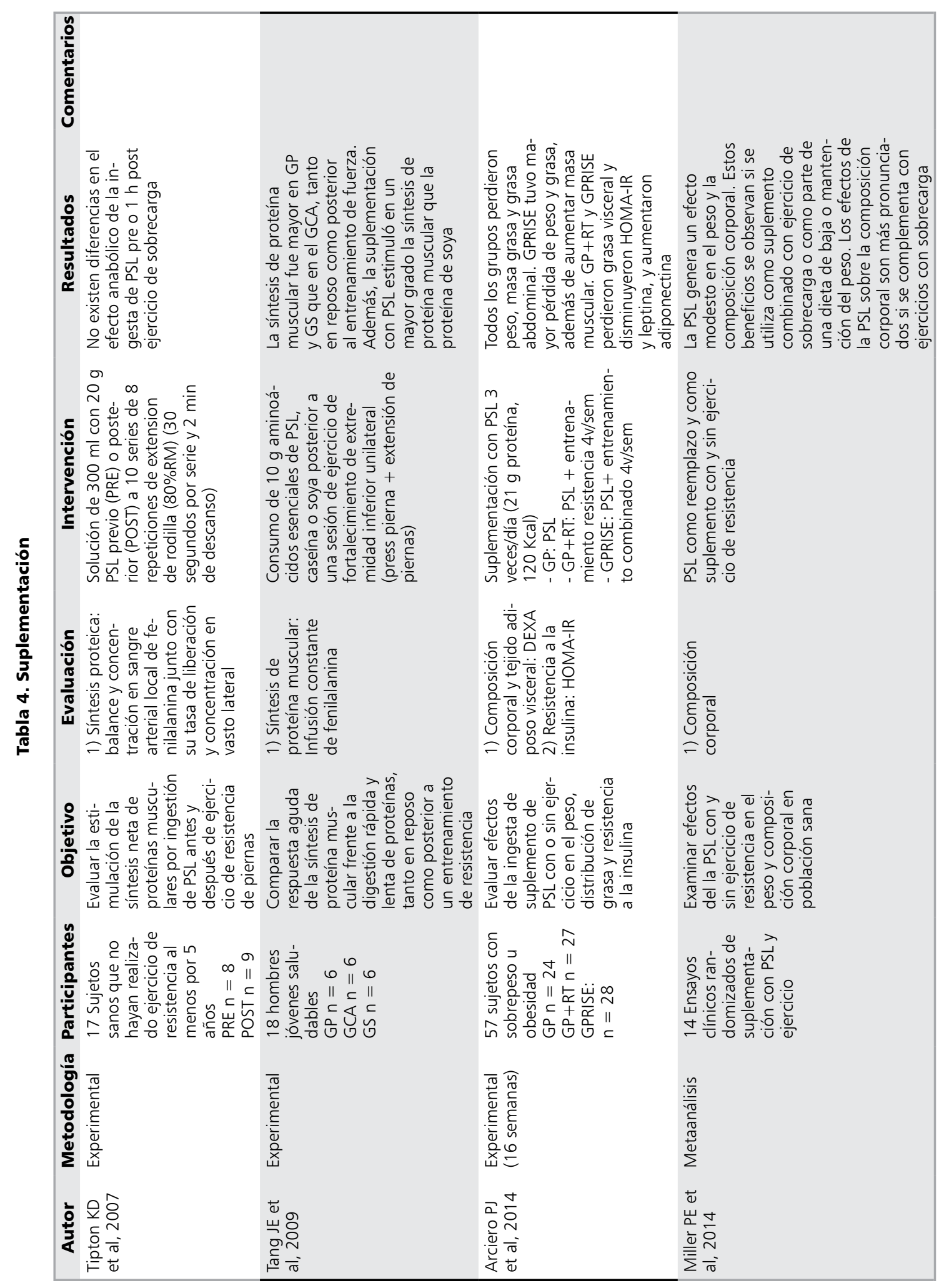




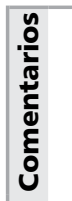

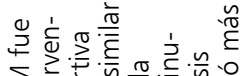

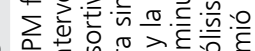

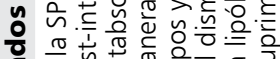

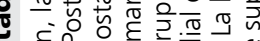

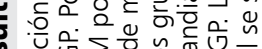

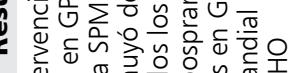

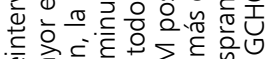

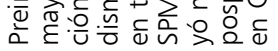

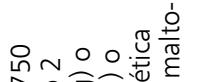

-

प.

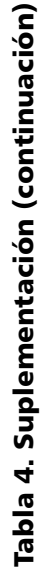

胥

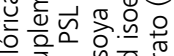

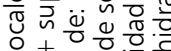

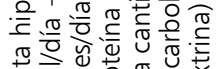

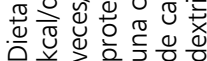

:을 :

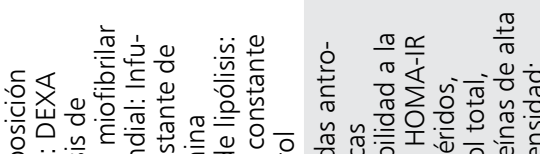

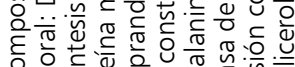
o 는

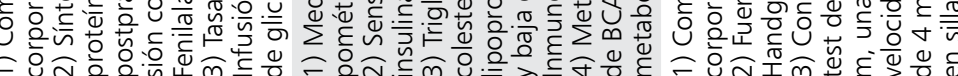

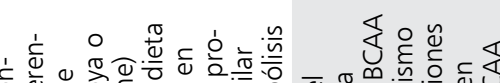

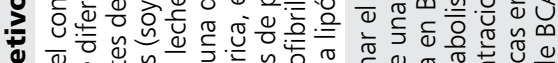

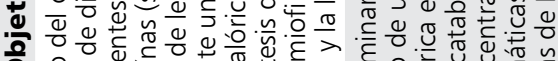

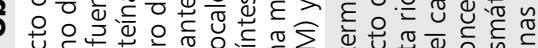

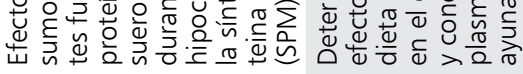

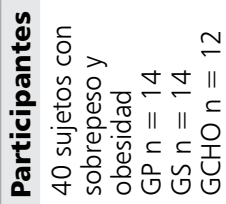

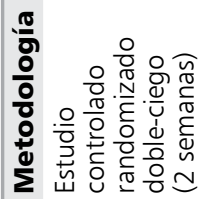

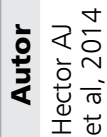
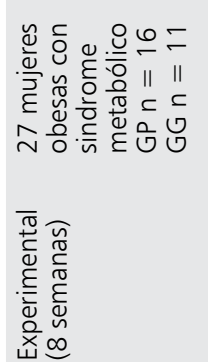

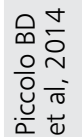

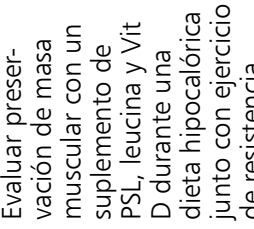
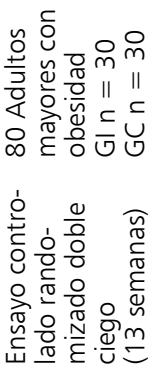

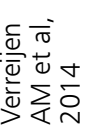

\section{$\vec{\Sigma} \cdot \frac{\pi}{\frac{\pi}{\pi}} \frac{\pi}{2}$

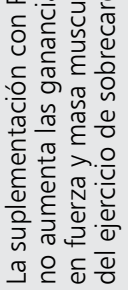

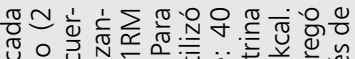

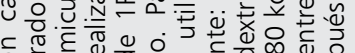

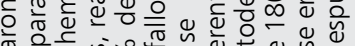

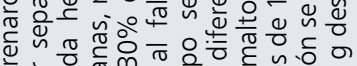

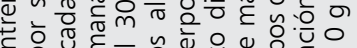

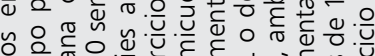

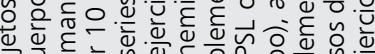

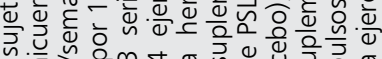
幺. है

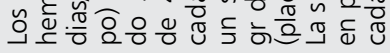

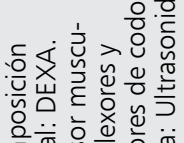

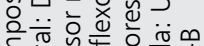

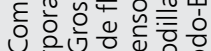

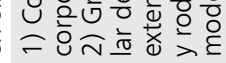

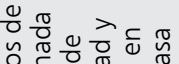

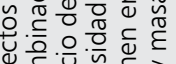
पथ हो 는

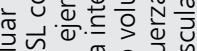

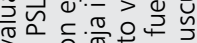

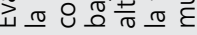

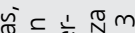

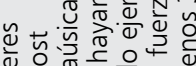
उ.

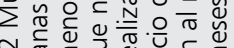

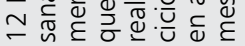

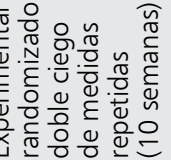

$\overline{\mathrm{s}}$

joi 


\begin{tabular}{|c|c|c|c|c|c|}
\hline & & 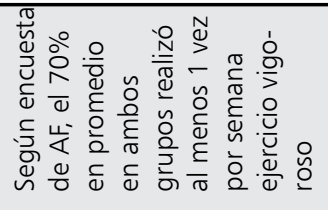 & 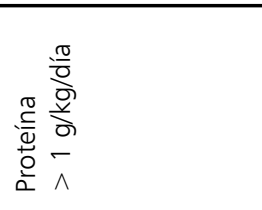 & 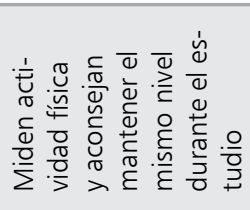 & 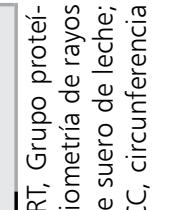 \\
\hline & 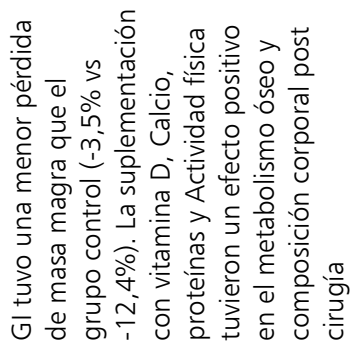 & 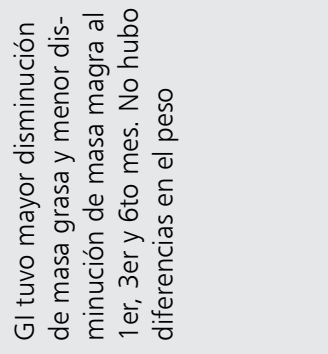 & 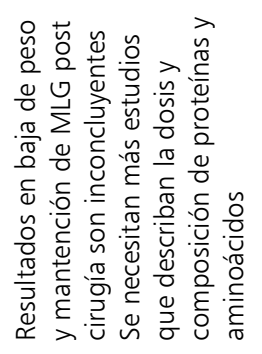 & 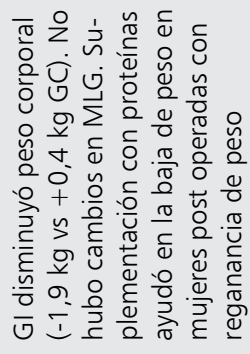 & 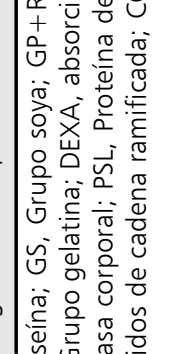 \\
\hline $\begin{array}{l}\text { 巳ు } \\
\underline{\underline{\Xi}}\end{array}$ & 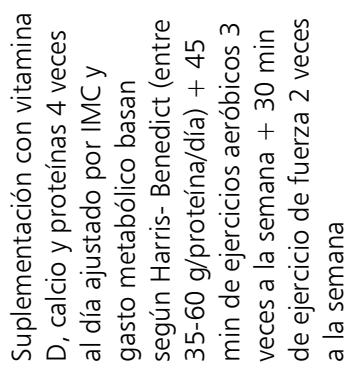 & 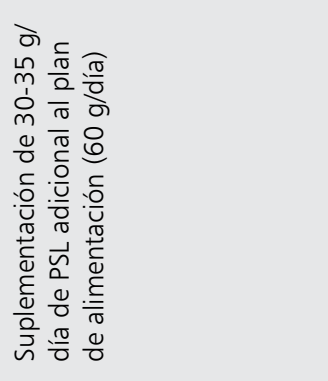 & 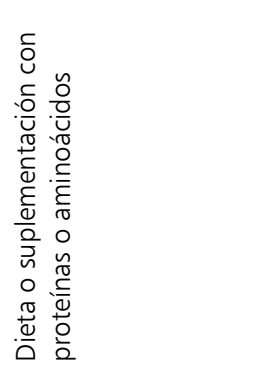 & 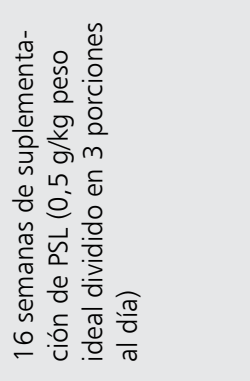 & 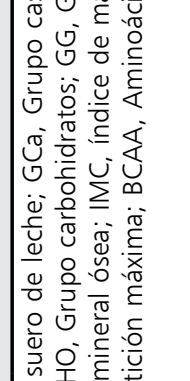 \\
\hline & 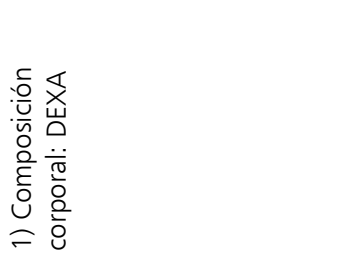 & 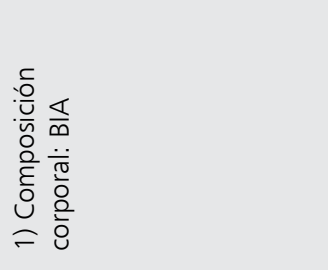 & 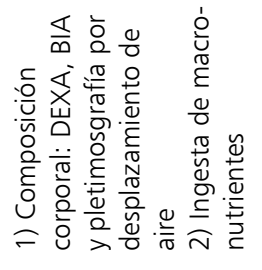 & 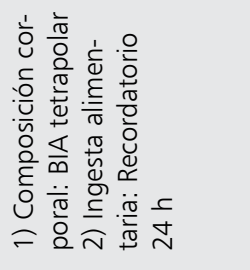 & 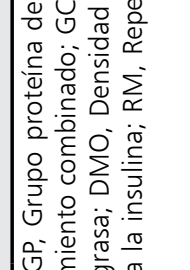 \\
\hline & 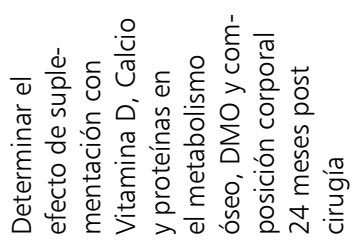 & 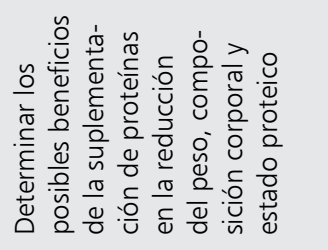 & 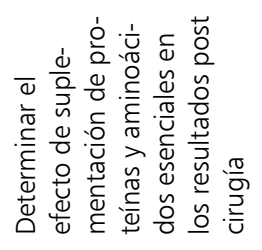 & 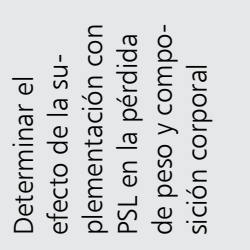 & 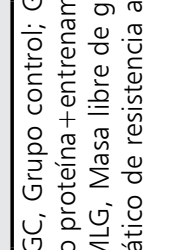 \\
\hline & 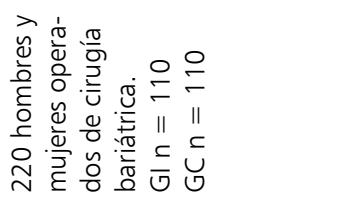 & 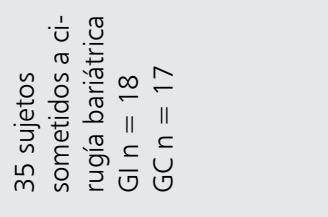 & 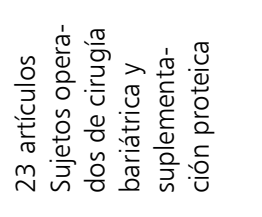 & 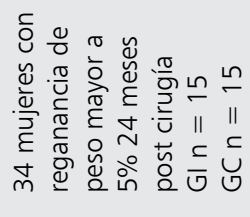 & 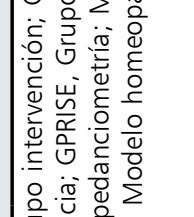 \\
\hline & 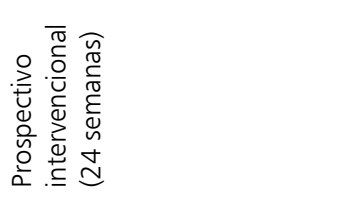 & 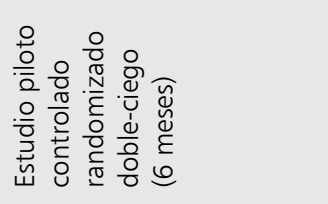 & 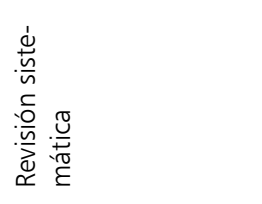 & 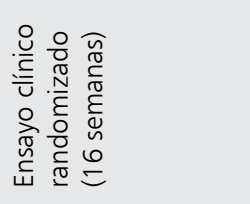 & $\frac{2}{3}$ \\
\hline गे & 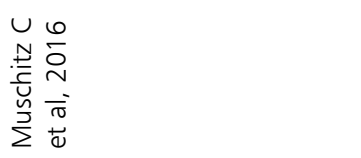 & 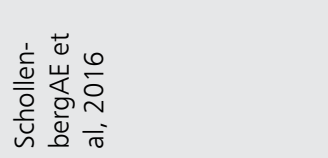 & 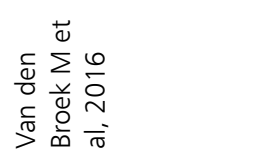 & 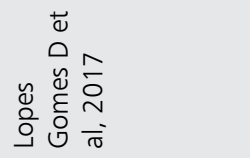 & 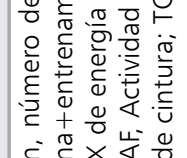 \\
\hline
\end{tabular}




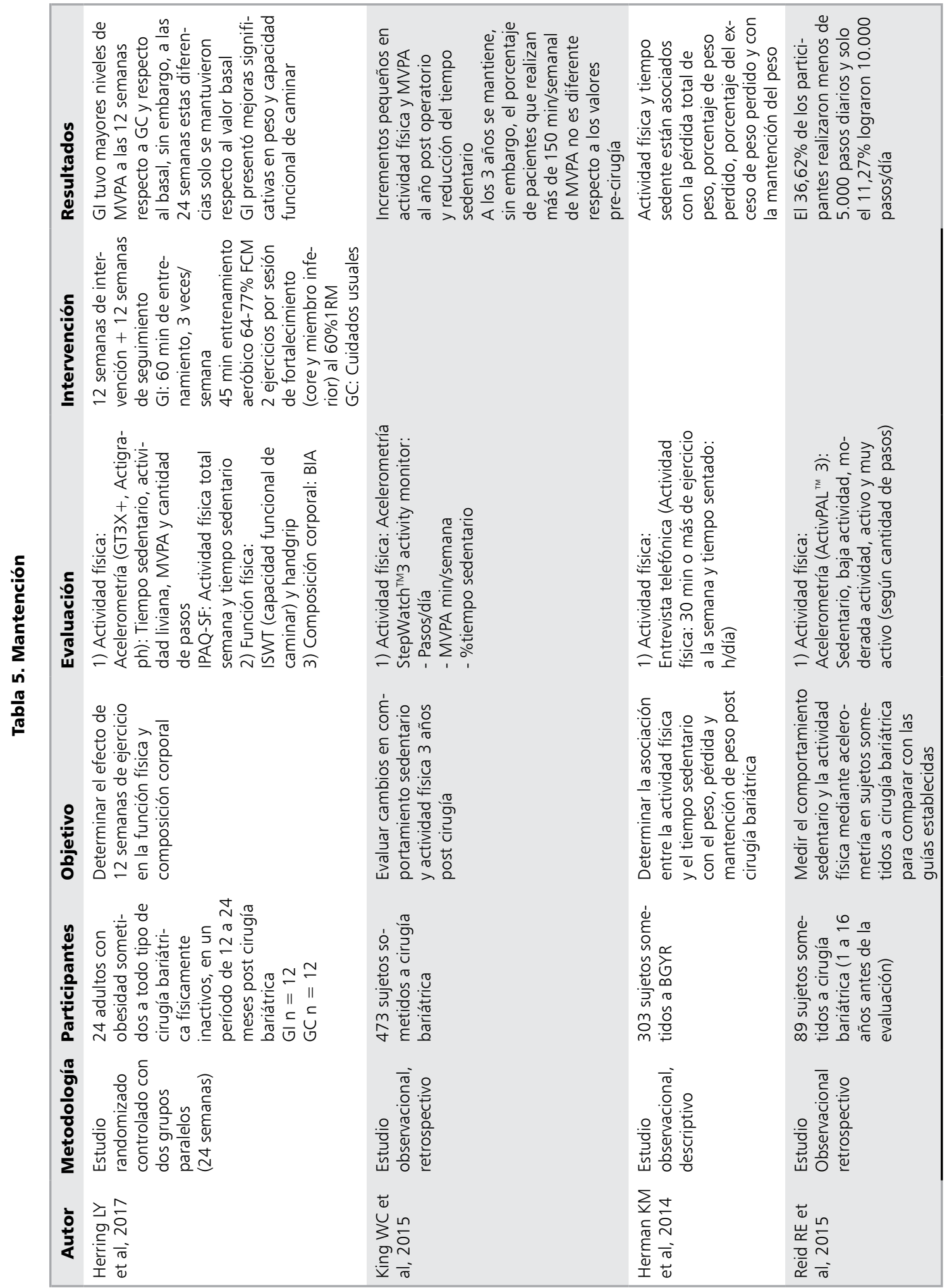




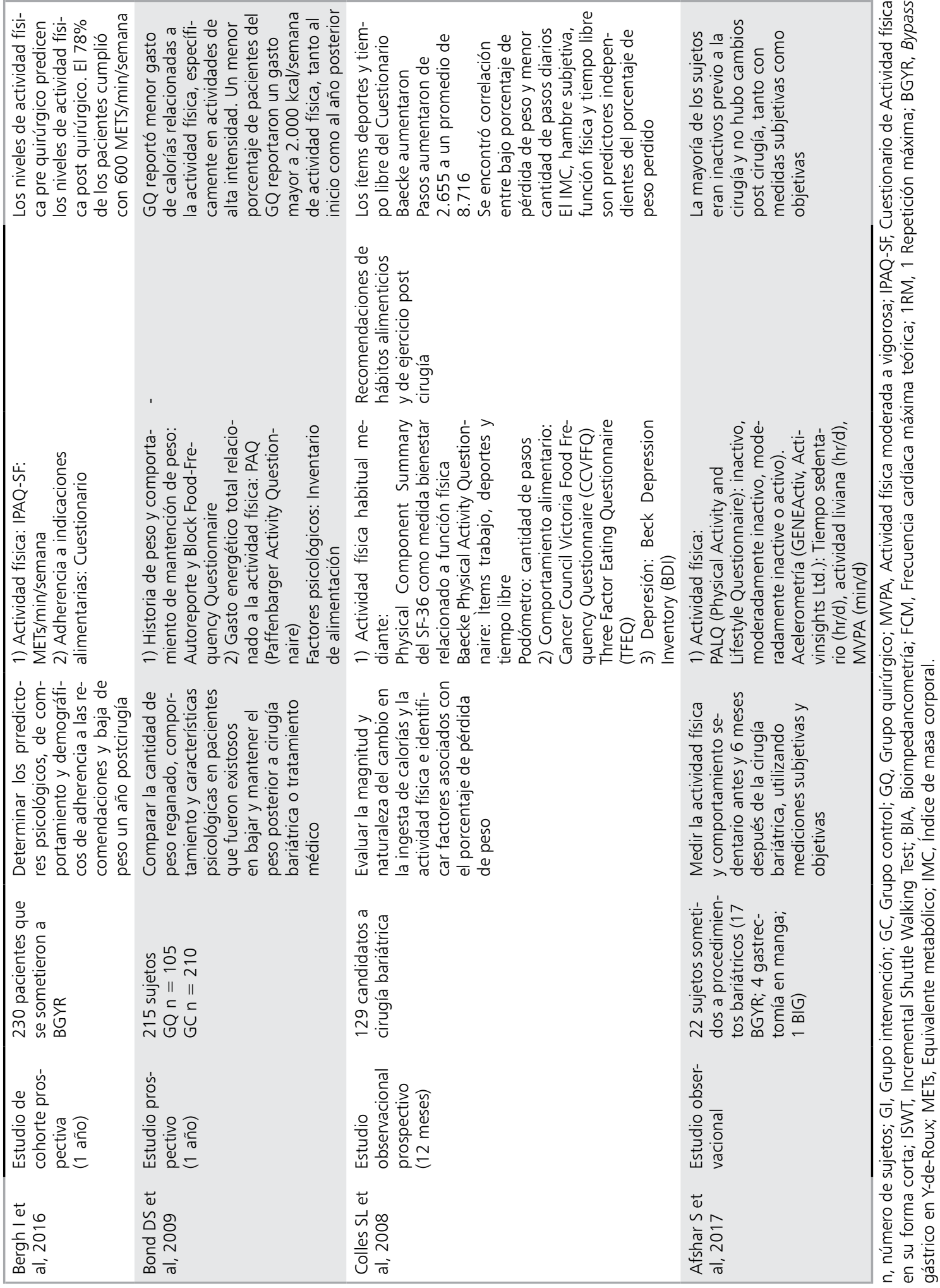


- En la fase de mantención del peso se recomienda realizar entre 200-300 min de actividad física moderada a vigorosa a la semana, siguiendo las recomendaciones del ACSM en relación a actividad física aeróbica, de fuerza y flexibilidad ${ }^{69}$. Es importante considerar que el incremento en los volúmenes debe ser progresivo para disminuir el riesgo e incidencia de lesiones asociadas a la realización de ejercicio ${ }^{70}$.

\section{Conclusión}

En conclusión, en este documento hemos propuesto lineamientos generales en la prescripción de ejercicio durante las diferentes etapas de la cirugía. No obstante, siempre debe realizarse un cuidadoso análisis de cada caso tomando en cuenta los antecedentes clínicos personales, además del efecto descrito para cada tipo de ejercicio y sus respectivas dosificaciones. El ejercicio en el paciente bariátrico es un tema que aún se encuentra en desarrollo por lo que es fundamental mantenerse al pendiente de futuras publicaciones y realizar más investigaciones en la población chilena.

\section{Referencias}

1. Ng M, Fleming T, Robinson M, Thomson B, Graetz N, Margono C, et al. Global, regional, and national prevalence of overweight and obesity in children and adults during 1980-2013: a systematic analysis for the Global Burden of Disease Study 2013. Lancet 2014; 384 (9945): 766-81.

2. Nguyen NT, Varela JE. Bariatric surgery for obesity and metabolic disorders: state of the art. Nat Rev Gastroenterol Hepatol 2017; 14 (3): 160-9.

3. Coen PM, Goodpaster BH. A role for exercise after bariatric surgery? Diabetes Obes Metab 2016; 18 (1): 16-23.

4. Busetto L, Dicker D, Azran C, Batterham RL, Farpour-Lambert N, Fried M, et al. Obesity Management Task Force of the European Association for the Study of Obesity Released "Practical Recommendations for the Post-Bariatric Surgery Medical Management”. Obes Surg 2018; 28 (7): 2117-21.

5. Livhits M, Mercado C, Yermilov I, Parikh JA, Dutson E, Mehran A, et al. Exercise following bariatric surgery: systematic review. Obes Surg 2010; 20 (5): 657-65.

6. Hassannejad A, Khalaj A, Mansournia MA, Rajabian
Tabesh M, Alizadeh Z. The Effect of Aerobic or Aerobic-Strength Exercise on Body Composition and Functional Capacity in Patients with BMI $>/=35$ after Bariatric Surgery: a Randomized Control Trial. Obes Surg 2017; 27 (11): 2792-801.

7. Iberoamericano CC. Manual Cochrane de revisiones sistemáticas de intervenciones, versión 5.1. 0. Barcelona: entro Cochrane Iberoamericano. 2012.

8. Peacock JC, Sloan SS, Cripps B. A qualitative analysis of bariatric patients' post-surgical barriers to exercise. Obes Surg 2014; 24 (2): 292-8.

9. Shah M, Snell PG, Rao S, Adams-Huet B, Quittner C, Livingston $\mathrm{EH}$, et al. High-volume exercise program in obese bariatric surgery patients: a randomized, controlled trial. Obesity (Silver Spring) 2011; 19 (9): 1826-34.

10. Coen PM, Menshikova EV, Distefano G, Zheng D, Tanner CJ, Standley RA, et al. Exercise and Weight Loss Improve Muscle Mitochondrial Respiration, Lipid Partitioning, and Insulin Sensitivity After Gastric Bypass Surgery. Diabetes 2015; 64 (11): 3737-50.

11. Coen PM, Tanner CJ, Helbling NL, Dubis GS, Hames $\mathrm{KC}$, Xie $\mathrm{H}$, et al. Clinical trial demonstrates exercise following bariatric surgery improves insulin sensitivity. J Clin Invest 2015; 125 (1): 248-57.

12. Woodlief TL, Carnero EA, Standley RA, Distefano G, Anthony SJ, Dubis GS, et al. Dose response of exercise training following roux-en-Y gastric bypass surgery: A randomized trial. Obesity (Silver Spring) 2015; 23 (12): 2454-61.

13. Baillot A, Mampuya WM, Dionne IJ, Comeau E, Meziat-Burdin A, Langlois MF. Impacts of Supervised Exercise Training in Addition to Interdisciplinary Lifestyle Management in Subjects Awaiting Bariatric Surgery: a Randomized Controlled Study. Obes Surg 2016; 26 (11): 2602-10.

14. Cummings DE, Arterburn DE, Westbrook EO, Kuzma JN, Stewart SD, Chan CP, et al. Gastric bypass surgery vs intensive lifestyle and medical intervention for type 2 diabetes: the CROSSROADS randomised controlled trial. Diabetologia 2016; 59 (5): 945-53.

15. Browning MG, Franco RL, Herrick JE, Arrowood JA, Evans RK. Assessment of Cardiopulmonary Responses to Treadmill Walking Following Gastric Bypass Surgery. Obes Surg 2017; 27 (1): 96-101.

16. Carnero EA, Dubis GS, Hames KC, Jakicic JM, Houmard JA, Coen PM, et al. Randomized trial reveals that physical activity and energy expenditure are associated with weight and body composition after RYGB. Obesity 2017; 25 (7): 1206-16.

17. Herring LY, Stevinson C, Carter P, Biddle SJH, Bowrey $\mathrm{D}$, Sutton C, et al. The effects of supervised exercise 
training 12-24 months after bariatric surgery on physical function and body composition: a randomised controlled trial. Int J Obes (Lond) 2017; 41 (6): 909-16.

18. Nunez López YO, Coen PM, Goodpaster BH, Seyhan AA. Gastric bypass surgery with exercise alters plasma microRNAs that predict improvements in cardiometabolic risk. Int J Obes (Lond) 2017; 41 (7): 1121-30.

19. Medicine ACoS. ACSM's health-related physical fitness assessment manual: Lippincott Williams \& Wilkins; 2013.

20. Pouwels S, Smelt HJM, Smulders JF. The Underestimated Effect of Perioperative Exercise Interventions in Bariatric Surgery: Increasing Need for Large Impact Studies. Obes Surg 2017; 27 (10): 2690-1.

21. da Silva RP, Martínez D, Faria CC, de Carli LA, de Souza WI, Meinhardt NG, et al. Improvement of exercise capacity and peripheral metaboreflex after bariatric surgery. Obes Surg 2013; 23 (11): 1835-41.

22. Tanaka H, Monahan KD, Seals DR. Age-predicted maximal heart rate revisited. J Am Coll Cardiol 2001; 37 (1): 153-6.

23. King WC, Chen JY, Bond DS, Belle SH, Courcoulas AP, Patterson EJ, et al. Objective assessment of changes in physical activity and sedentary behavior: Pre- through 3 years post-bariatric surgery. Obesity (Silver Spring). 2015; 23 (6): 1143-50.

24. Daniels P, Burns RD, Brusseau TA, Hall MS, Davidson L, Adams TD, et al. Effect of a randomised 12-week resistance training programme on muscular strength, cross-sectional area and muscle quality in women having undergone Roux-en-Y gastric bypass. J Sports Sci 2018; 36 (5): 529-35.

25. Medicine ACoS. ACSM's guidelines for exercise testing and prescription: Lippincott Williams \& Wilkins; 2013.

26. Desgorces FD, Berthelot G, Dietrich G, Testa MS. Local muscular endurance and prediction of 1 repetition maximum for bench in 4 athletic populations. J Strength Cond Res 2010; 24 (2): 394-400.

27. Neunhaeuserer D, Gasperetti A, Savalla F, Gobbo S, Bullo V, Bergamin M, et al. Functional Evaluation in Obese Patients Before and After Sleeve Gastrectomy. Obes Surg 2017; 27 (12): 3230-9.

28. Nikseresht M. Comparison of Serum Cytokine Levels in Men Who are Obese or Men Who are Lean: Effects of Nonlinear Periodized Resistance Training and Obesity. J Strength Cond Res 2018; 32 (6): 1787-95.

29. Gallup AC, Fink B. Handgrip Strength as a Darwinian Fitness Indicator in Men. Front Psychol 2018; 9: 439.

30. Gomes DL, de Almeida Oliveira D, Dutra ES, Pizato N, de Carvalho KM. Resting Energy Expenditure and Body Composition of Women with Weight Regain 24
Months After Bariatric Surgery. Obes Surg 2016; 26 (7): 1443-7.

31. Vink RG, Roumans NJ, Arkenbosch LA, Mariman EC, van Baak MA. The effect of rate of weight loss on long-term weight regain in adults with overweight and obesity. Obesity (Silver Spring) 2016; 24 (2): 321-7.

32. Roman M, Monaghan A, Serraino GF, Miller D, Pathak S, Lai F, et al. Meta-analysis of the influence of lifestyle changes for preoperative weight loss on surgical outcomes. Br J Surg 2019; 106 (3): 181-9.

33. Donnelly J, Blair S, Jakicic J, Manore M, Rankin J, Smith B. Appropriate Physical Activity Intervention Strategies for Weight Loss and Prevention of Weight Regain for Adults. Medicine and Science in Sports and Exercise 2009; 41 (2): 459-71.

34. Giusti V, Theytaz F, Di Vetta V, Clarisse M, Suter M, Tappy L. Energy and macronutrient intake after gastric bypass for morbid obesity: a 3-y observational study focused on protein consumption. Am J Clin Nutr 2016; 103 (1): 18-24.

35. Tipton KD, Elliott TA, Cree MG, Aarsland AA, Sanford AP, Wolfe RR. Stimulation of net muscle protein synthesis by whey protein ingestion before and after exercise. Am J Physiol Endocrinol Metab 2007; 292 (1): E71-6.

36. Tang JE, Moore DR, Kujbida GW, Tarnopolsky MA, Phillips SM. Ingestion of whey hydrolysate, casein, or soy protein isolate: effects on mixed muscle protein synthesis at rest and following resistance exercise in young men. J Appl Physiol (1985) 2009; 107 (3): 987-92.

37. Arciero PJ, Baur D, Connelly S, Ormsbee MJ. Timed-daily ingestion of whey protein and exercise training reduces visceral adipose tissue mass and improves insulin resistance: the PRISE study. J Appl Physiol (1985) 2014; 117 (1): 1-10.

38. Miller PE, Alexander DD, Pérez V. Effects of whey protein and resistance exercise on body composition: a meta-analysis of randomized controlled trials. J Am Coll Nutr 2014; 33 (2): 163-75.

39. Hector AJ, Marcotte GR, Churchward-Venne TA, Murphy $\mathrm{CH}$, Breen L, von Allmen M, et al. Whey protein supplementation preserves postprandial myofibrillar protein synthesis during short-term energy restriction in overweight and obese adults. J Nutr 2015; 145 (2): 246-52.

40. Piccolo BD, Comerford KB, Karakas SE, Knotts TA, Fiehn O, Adams SH. Whey Protein Supplementation Does Not Alter Plasma Branched-Chained Amino Acid Profiles but Results in Unique Metabolomics Patterns in Obese Women Enrolled in an 8-Week Weight Loss Trial-4. The Journal of nutrition 2015; 145 (4): 691-700. 
41. Verreijen AM, Verlaan S, Engberink MF, Swinkels S, de Vogel-van den Bosch J, Weijs PJ. A high whey protein-, leucine-, and vitamin D-enriched supplement preserves muscle mass during intentional weight loss in obese older adults: a double-blind randomized controlled trial. Am J Clin Nutr 2015; 101 (2): 279-86.

42. Weisgarber KD, Candow DG, Farthing JP. Whey protein and high-volume resistance training in postmenopausal women. J Nutr Health Aging 2015; 19 (5): 511-7.

43. Muschitz C, Kocijan R, Haschka J, Zendeli A, Pirker T, Geiger C, et al. The Impact of Vitamin D, Calcium, Protein Supplementation, and Physical Exercise on Bone Metabolism After Bariatric Surgery: The BABS Study. J Bone Miner Res 2016; 31 (3): 672-82.

44. Schollenberger AE, Karschin J, Meile T, Kuper MA, Konigsrainer A, Bischoff SC. Impact of protein supplementation after bariatric surgery: A randomized controlled double-blind pilot study. Nutrition 2016; 32 (2): 186-92.

45. van den Broek M, de Heide LJ, Veeger NJ, van der WalOost AM, van Beek AP. Influence of dietary protein and its amino acid composition on postoperative outcomes after gastric bypass surgery: a systematic review. Nutr Rev 2016; 74 (12): 749-73.

46. Lopes Gomes D, Moehlecke M, Lopes da Silva FB, Dutra ES, D'Agord Schaan B, Baiocchi de Carvalho KM. Whey Protein Supplementation Enhances Body Fat and Weight Loss in Women Long After Bariatric Surgery: a Randomized Controlled Trial. Obes Surg 2017; 27 (2): 424-31.

47. Layman DK, Walker DA. Potential importance of leucine in treatment of obesity and the metabolic syndrome. J Nutr 2006; 136 (1 Suppl): 319S-23S.

48. Luhovyy BL, Akhavan T, Anderson GH. Whey proteins in the regulation of food intake and satiety. J Am Coll Nutr 2007; 26 (6): 704S-12S.

49. Pal S, Radavelli-Bagatini S. The effects of whey protein on cardiometabolic risk factors. Obes Rev 2013; 14 (4): 324-43.

50. Cribb PJ, Hayes A. Effects of supplement timing and resistance exercise on skeletal muscle hypertrophy. Med Sci Sports Exerc 2006; 38 (11): 1918-25.

51. Bond DS, Jakicic JM, Vithiananthan S, Thomas JG, Leahey TM, Sax HC, et al. Objective quantification of physical activity in bariatric surgery candidates and normal-weight controls. Surg Obes Relat Dis 2010; 6 (1): 72-8.

52. Bond DS, Unick JL, Jakicic JM, Vithiananthan S, Pohl D, Roye GD, et al. Objective assessment of time spent being sedentary in bariatric surgery candidates. Obes Surg 2011; 21 (6): 811-4.

53. Unick JL, Bond DS, Jakicic JM, Vithiananthan S, Ry- der BA, Roye GD, et al. Comparison of two objective monitors for assessing physical activity and sedentary behaviors in bariatric surgery patients. Obes Surg 2012; 22 (3): 347-52.

54. Curioni CC, Lourenco PM. Long-term weight loss after diet and exercise: a systematic review. Int J Obes (Lond) 2005; 29 (10): 1168-74.

55. Bray GA. Diet and exercise for weight loss. JAMA 2012; 307 (24): 2641-2.

56. Bond DS, Phelan S, Leahey TM, Hill JO, Wing RR. Weight-loss maintenance in successful weight losers: surgical vs non-surgical methods. Int J Obes (Lond) 2009; 33 (1): 173-80.

57. King WC, Hsu JY, Belle SH, Courcoulas AP, Eid GM, Flum DR, et al. Pre- to postoperative changes in physical activity: report from the longitudinal assessment of bariatric surgery-2 (LABS-2). Surg Obes Relat Dis 2012; 8 (5): 522-32.

58. Herman KM, Carver TE, Christou NV, Andersen RE. Keeping the weight off: physical activity, sitting time, and weight loss maintenance in bariatric surgery patients 2 to 16 years postsurgery. Obes Surg 2014; 24 (7): 1064-72.

59. Reid RE, Carver TE, Andersen KM, Court O, Andersen RE. Physical activity and sedentary behavior in bariatric patients long-term post-surgery. Obes Surg 2015; 25 (6): 1073-7.

60. Bergh I, Lundin Kvalem I, Risstad H, Sniehotta FF. Preoperative predictors of adherence to dietary and physical activity recommendations and weight loss one year after surgery. Surg Obes Relat Dis 2016; 12 (4): 910-8.

61. Bond DS, Phelan S, Wolfe LG, Evans RK, Meador JG, Kellum JM, et al. Becoming physically active after bariatric surgery is associated with improved weight loss and health-related quality of life. Obesity (Silver Spring). 2009; 17 (1): 78-83.

62. Colles SL, Dixon JB, O’Brien PE. Hunger control and regular physical activity facilitate weight loss after laparoscopic adjustable gastric banding. Obes Surg 2008; 18 (7): 833-40.

63. Afshar S, Seymour K, Kelly SB, Woodcock S, van Hees VT, Mathers JC. Changes in physical activity after bariatric surgery: using objective and self-reported measures. Surg Obes Relat Dis 2017; 13 (3): 474-83.

64. Tremblay MS, Warburton DE, Janssen I, Paterson DH, Latimer AE, Rhodes RE, et al. New Canadian physical activity guidelines. Appl Physiol Nutr Metab 2011; 36 (1): 36-46; 7-58.

65. Jensen MD, Ryan DH, Apovian CM, Ard JD, Comuzzie AG, Donato KA, et al. 2013 AHA/ACC/TOS guideline for the management of overweight and obesity in adults: 
a report of the American College of Cardiology/American Heart Association Task Force on Practice Guidelines and The Obesity Society. J Am Coll Cardiol 2014; 63 (25 Pt B): 2985-3023.

66. Swift DL, Johannsen NM, Lavie CJ, Earnest CP, Church TS. The role of exercise and physical activity in weight loss and maintenance. Prog Cardiovasc Dis 2014; 56 (4): 441-7.

67. Rutherford D. The Role of Physical Activity and Exercise in Managing Obesity and Achieving Weight Loss. Practical Guide to Obesity Medicine 2017; 215.

68. Tudor-Locke C, Craig CL, Aoyagi Y, Bell RC, Croteau KA, De Bourdeaudhuij I, et al. How many steps/day are enough? For older adults and special populations. Inter- national Journal of Behavioral Nutrition and Physical Activity 2011; 8 (1): 1.

69. Garber CE, Blissmer B, Deschenes MR, Franklin BA, Lamonte MJ, Lee IM, et al. American College of Sports Medicine position stand. Quantity and quality of exercise for developing and maintaining cardiorespiratory, musculoskeletal, and neuromotor fitness in apparently healthy adults: guidance for prescribing exercise. Med Sci Sports Exerc 2011; 43 (7): 1334-59.

70. Hootman JM, Macera CA, Ainsworth BE, Martin M, Addy CL, Blair SN. Association among physical activity level, cardiorespiratory fitness, and risk of musculoskeletal injury. American journal of epidemiology 2001; 154 (3): 251-8. 